\title{
Mirror-Augmented Photovoltaic Designs and Performance
}

\author{
Wei-Chun Lin, Dave Hollingshead, Kara A. Shell, Joseph Karas, Scott A. Brown, Mark Schuetz, \\ Yang $\mathrm{Hu}$, and Roger H. French, Member, IEEE
}

\begin{abstract}
In developing photovoltaic (PV) technology, it is crucial to provide lower cost $P V$ power. One of the useful methods is to increase power output of conventional modules since the major costs (module manufacturing, mounts, wiring, installation labor, etc) tend to scale with system area, and increased power output due to improved light harvesting will produce more power per unit area. Under this concept, our research group seeks to provide low cost power, using flat-panel PV modules, which have mirror augmented irradiance through the addition of low cost solar mirrors. In order to harvest more incident solar irradiance, an optimized design configuration between a flat-panel module and mirror are necessary for this fixed (non-tracked) mirror-augmented photovoltaic (MAPV) system. A series of MATLAB calculations were developed to screen various MAPV design configurations. TracePro is a ray tracing program for optical analysis of 3D solid models. We use TracePro to determine irradiance non-uniformity issues on the MAPV system. Both the Matlab and TracePro results are compared to outdoor field test results. I-V curve tracing of test modules is done with a Daystar Multi-tracer for time series analysis. Over a time-limited period of study the MAPV system produced $26.2 \%$ more power than an equivalent non-augmented panel.
\end{abstract}

Index Terms-Photovoltaic, MAPV, MATLAB, TracePro, Irradiance non-uniformity

\section{INTRODUCTION}

It is not easy for solar energy to compete with other low cost energy sources such as nuclear, thermal and wind power in geographic areas of lower solar insolation, such as the northern United States. One solution to make solar energy competitive is to combine reflectors with the PV modules in the system. Using solar mirrors to harvest more of the incident solar irradiance and direct sunlight to qualified PV modules increases the electricity produced from a given number or area of PV panels ${ }^{1}$. Replex Plastics has developed a high performance, low-cost solar mirror made of acrylic ${ }^{2}$. The acrylic mirror uses a back surface reflector and is ideal specifically for PV augmentation because of its light weight and low light scattering. In addition, the solar mirror, due to its back surface reflector, can be designed with UV absorbing

This work was supported by the Ohio Third Frontier under Tech 11-060 and Tech 12-004.

W. C. Lin, J. Karas, Y. Hu and R. H. French are with the Department of Materials Science and Engineering, Case Western Reserve University, Cleveland, $\mathrm{OH} 44106$ USA (e-mail:wxl157@case.edu, rxf131@case.edu )

D. Hollingshead, K. A. Shell, S. A. Brown, and M. A. Schuetz are with Replex Plastics, Mount Vernon, $\mathrm{OH} 43050$ USA (e-mail: dave@replex.com ). and UV reflecting properties which can prevent the PV panel from experiencing additional harmful UV irradiance reflected from the mirror. Therefore, MAPV systems can improve harvesting of the fixed $1 \mathrm{~kW} / \mathrm{m}^{2}$ solar resource, and make solar energy competitive with other energy systems.

\section{TECHNICAL APPROACH}

In order to obtain maximized power output for a location, the next topic is to optimize the mirror and module configuration via simulation in MATLAB. For this calculation we will optimize the total annual incident power on the mirror augmented module (the MAPV system) at any given time. It is noteworthy that for fixed MAPV systems (i.e. that don't use trackers to orient the MAPV system with the sun) flat mirrors reflect light onto the PV panel creating discrete "bands" of illumination across the panel, and the band intensity and position depend on two important parameters: sun position and mirror \& module tilt angles. The sun position calculation for every minute for the given installation location is based on a technical report developed by Reda and Andreas in NREL ${ }^{3}$. The longitude and latitude were set to Columbus, Ohio. No cloud cover or weather is considered and no edge effects are included because mirrors are assumed as infinitely long. The MATLAB modeling starts from setting a specific tilt angle for the module and mirror, for example, panel tilt at $50^{\circ}$ and mirror tilt at $10^{\circ}$. Then, the sun position for a given installation location is computed and the power density of the light (in $\mathrm{kW} / \mathrm{m}^{2}$ ) that hits the panel at a given instant in time (e.g. 7:45am on January $1^{\text {st }}$ ) is found. The incident illumination on module and mirrors is based on sun's azimuth and elevation angle. This process is repeated for the entire year to calculate the cumulative amount of annual incident power. From the description above, we can estimate the amount of power for a specific model design. Further calculations can be performed for different system designs (e.g panel tilt $=35^{\circ}$, mirror tilt $=0^{\circ}$ ) by simply repeating the above process.

In addition to absolute power density, to obtain an optimum model configuration, the irradiance distribution on PV modules must be studied. Since the fixed PV panels were augmented by mirrors throughout the day, the occurrence of non-uniform irradiance distribution on modules is expected. The non-uniformity issue is an important factor to fully study power production capability and estimate degradation rates. PV modules which receive highly localized irradiance may experience faster degradation rates. TracePro, developed by 
Lambda Research Corp. was used to observe the light distribution on the module. This software allows us to complete detailed ray trace analysis on a model without making any assumptions as to the order in which objects and surfaces will be intersected. At each intersection, individual rays can be subject to absorption, reflection, refraction, diffraction and scatter. Furthermore, TracePro can construct solid models geometrically and it is also compatible with other computer aided design (CAD) programs such as SolidWorks. The models will be constructed and analyzed in TracePro based on the MATLAB optimum results.

Finally, an outdoor test facility has been constructed at Replex Plastics for real-world data collection. The I-V curves are collected from a 220W Canadian Solar Inc. (CSI) CS-6P module $^{4}$ using a Daystar Multi-Tracer ${ }^{5}$.

\section{MODEL CONFIGURATION}

\section{A. MATLAB Modeling}

As mentioned above, model configuration should be determined prior to optical analysis and outdoor field test. The MATLAB model was used to estimate the illumination pattern on a mirror augmented module and to optimize mirror and module configuration for maximized annual power output. Figure 1 shows the normalized irradiance result versus mirror and module tilt angle for possible system combinations. It is observed that the system receives maximum irradiance (the climax of green line) when the module tilt angle $=50^{\circ}$ and mirror tilt angle $=10^{\circ}$ from the horizontal. We choose this module and mirror tilt $\left(50^{\circ}\right.$ and $10^{\circ}$ respectively) as our configuration for further analysis.

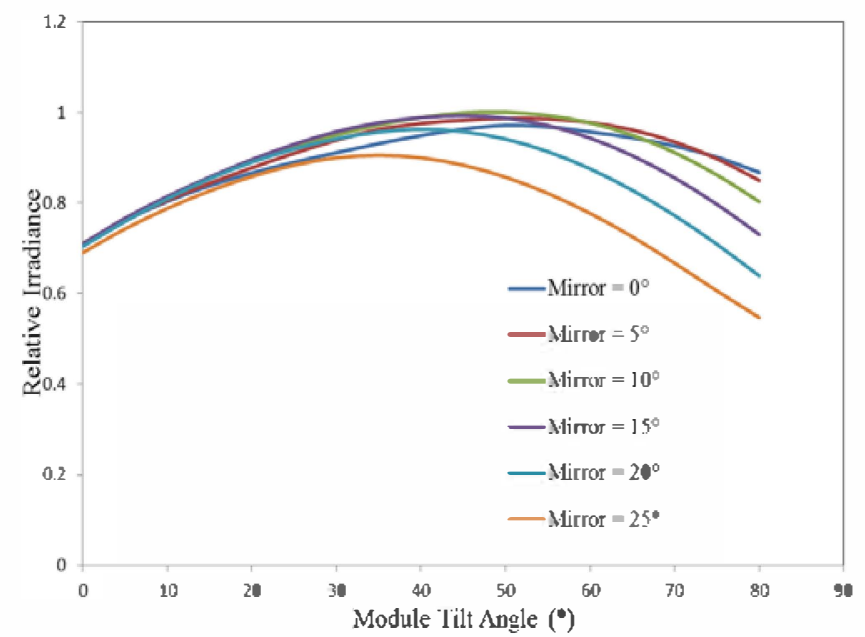

Figure 1. Relative irradiance vs. module tilt for a number of mirror tilt angles.

\section{B. Optical Analysis}

The optical analysis starts with the creation of a solid computer aided design (CAD) model representing the configuration designed in the previous section. Figure 2 illustrates two such models which are used for further analysis. Figure 2a shows the model with a mirror matching the panel length and the $\mathrm{z}$-axis represents the north direction. Figure $\mathbf{2 b}$ shows the model with a $3 \mathrm{x}$ wide mirror in order to remove the end-effect on the panel that may be seen at high incidence angles. The $\mathrm{x}$-axis represents the east direction. The second step is to import the models into TracePro and to define material and surface properties for the appropriate objects in the model. The material and surface properties representing desired attributes such as reflection and absorption are defined for our geometrical model. The next step is the simulation process in which the rays are traced from a grid light source onto the defined model. The final step is to analyze the ray trace results to determine the location, extent and distribution of the resultant illumination on the panel and mirrors.

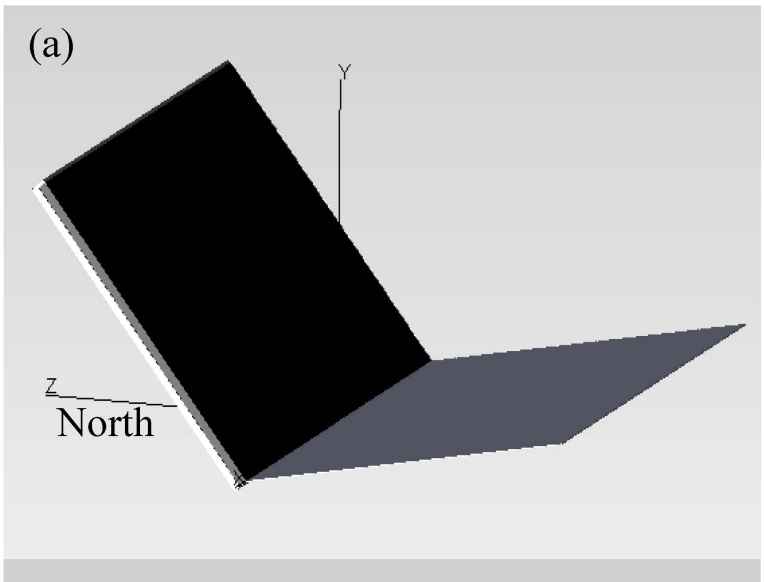

(b)

Figure 2. Two models will be used for optical analysis: model with (a) mirror matching panel, and (b) $3 x$ wide mirror.

\section{Outdoor Test Facility Construction}

The outdoor MAPV configuration is based on the MATLAB modeling result. A row of three consecutive PV panels (CSI CS-6P) was coupled with Replex back surface acrylic solar mirrors. A second row of non-augmented modules was also installed to enable simultaneous side-byside comparison between augmented and non-augmented modules. Figure 3 demonstrates the MAPV construction installed at Replex test site near Columbus, OH. For the I-V curve investigation, the primary instrument used is a Daystar Multi-Tracer. This device is a self-contained load and data acquisition device for collecting PV module performance. The 
multi-tracer also collects input from auxiliary devices measuring irradiance and temperature, which can be used to normalize power measurements and $\mathrm{I}-\mathrm{V}$ curves to standard temperature and conditions (STC). Contact thermocouples and a solar pyranometer ${ }^{6}$ were obtained and calibrated for this purpose.

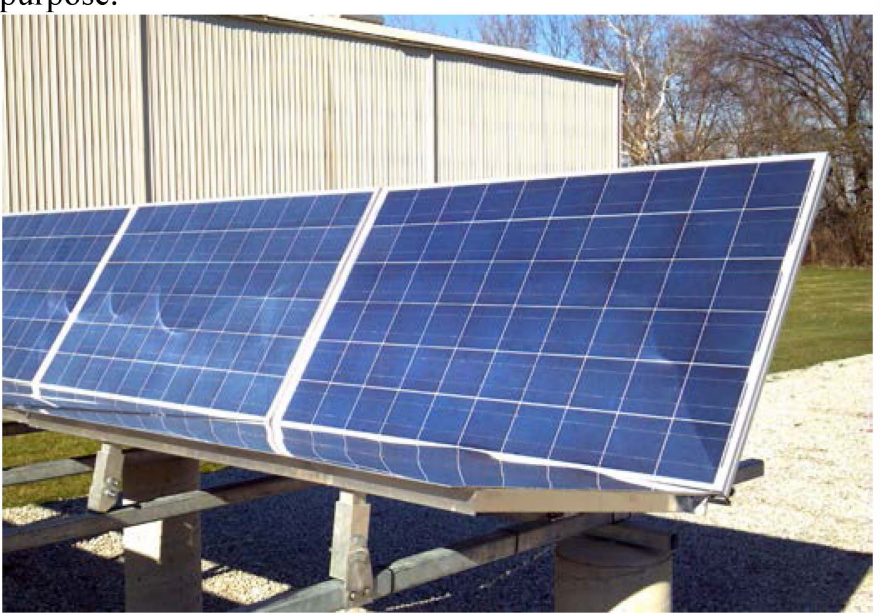

Figure 3. The MAPV test system at Replex Plastics.

\section{RESUlTS AND DISCUSSION}

\section{A. Ray-Tracing Simulation}

In this model, the longitude and latitude of the sun was assumed in Cleveland. Figure $\mathbf{4}$ shows the irradiance distribution of MAPV with the mirror matching to PV panel from $8 \mathrm{am}$ to $4 \mathrm{pm}$ (model shown in Figure 2a). It can be observed that the mirror augmentation causes the nonuniformity issue mostly shown on the right side panel at each time in figure 4. The irradiance map shows non-uniformity caused by the mirror shadow effect at $8 \mathrm{am}$. The sunlight cannot hit on a small region (upper right black region in figure 4 at 8am) of the panel because the mirror is blocking the solar irradiance at these times. The lower irradiance value region on the upper right side of $8 \mathrm{am}$ represents the bottom region on the west side of panel as shown in Figure 2a. The irradiance maps at $9 \mathrm{am}$ and $4 \mathrm{pm}$ show that the panels were fully illuminated and the mirror did not add illumination on the PV panel. Hence, two uniform irradiance maps were obtained. From $10 \mathrm{am}$ to $3 \mathrm{pm}$, the non-uniformity caused by mirror augmentation occurs at the bottom region of model. The augmented bottom region of model also shows that the reflected light does not hit uniformly on the panel. It indicates that a small region at the corner is not augmented by the mirror. This is due to the end-effect created by the short mirror length. Furthermore, it is observed that the irradiance maps are symmetric about noon from $10 \mathrm{am}$ to $3 \mathrm{pm}$ because of the similar elevation angles of sun.

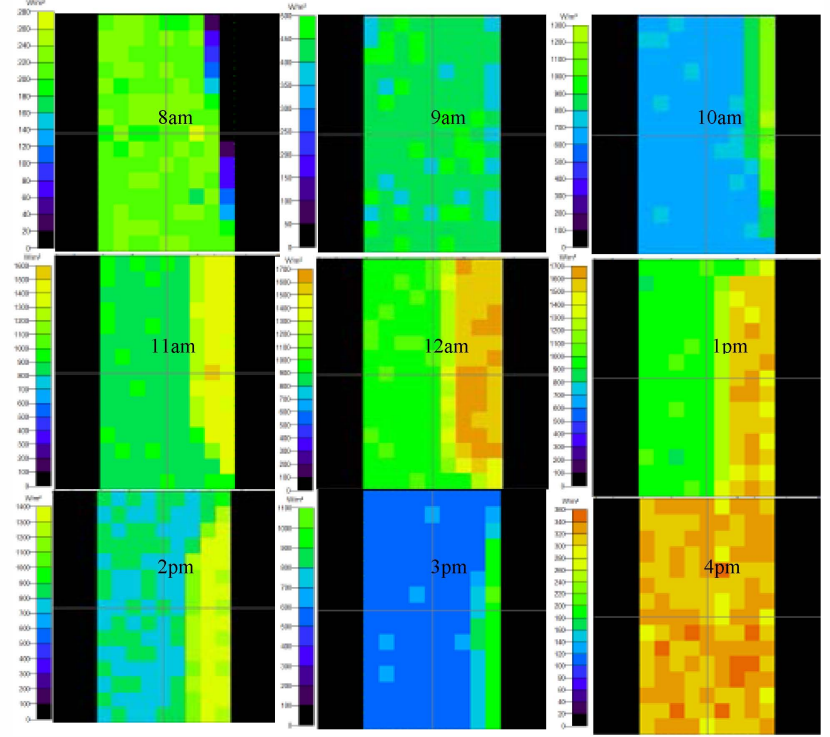

Figure 4. Irradiance maps of MAPV model with short mirror from $8 \mathrm{am}$ to $4 \mathrm{pm}$.

In order to remove the end-effect, a $3 \mathrm{x}$ wide mirror was designed to obtain higher uniformity for morning and afternoon irradiance. Figure 5 shows the irradiance maps of MAPV model with longer mirror. At 8am, the bottom region of panel cannot receive any irradiance because of shadow effect by the $3 \mathrm{x}$ wide mirror. The irradiance maps also present uniform distribution at $9 \mathrm{am}$ and $4 \mathrm{pm}$. The irradiance maps show a symmetrically non-uniform phenomenon from $10 \mathrm{am}$ to $3 \mathrm{pm}$ caused by mirror augmentation. It is similar to previous model but removing the end-effect by $3 \mathrm{x}$ wide mirror.

Figure 6 shows the average irradiance value of nonaugmented PV and MAPV models. It is shown that the irradiance value can be increased by the mirror up to almost $20 \%$ at noon. The irradiance values of $3 \mathrm{x}$ wide model are a little bit higher than the model with short mirror.

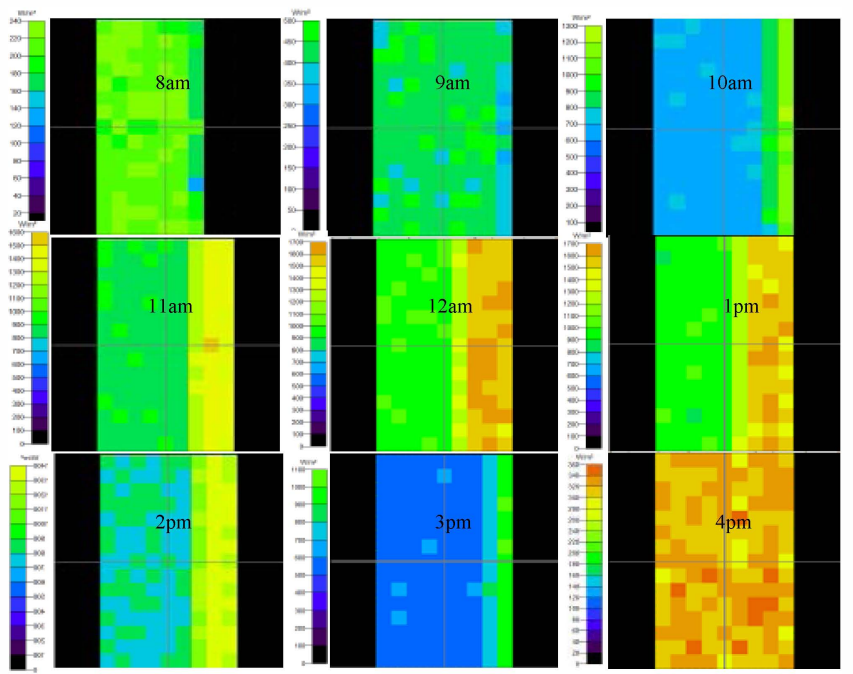

Figure 5. Irradiance maps of MAPV model with $3 \mathrm{x}$ wide mirror from $8 \mathrm{am}$ to $4 \mathrm{pm}$. 


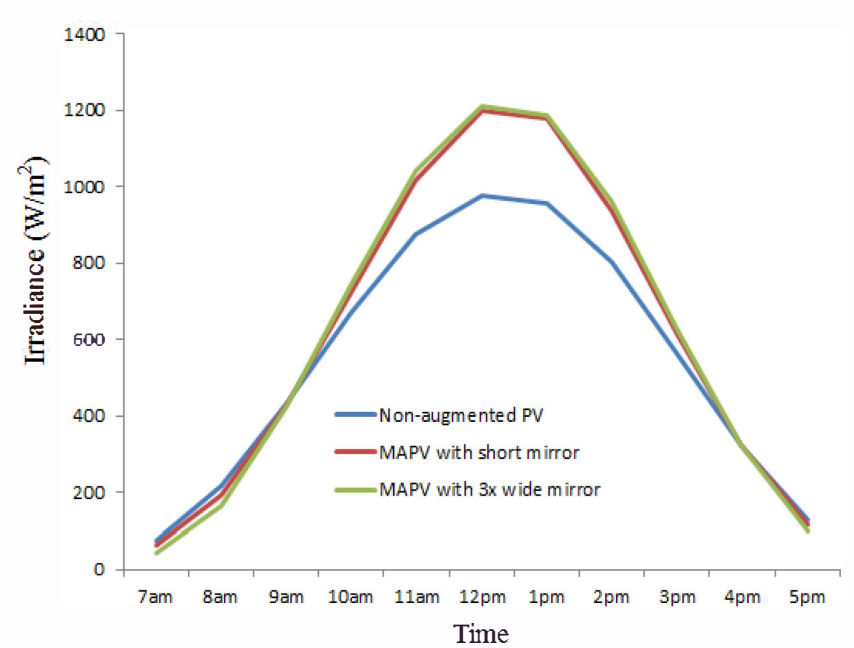

Figure 6. The irradiance of non-augmented PV and MAPV models.

\section{B. Field Test Result}

Figure 7 compares the I-V curves of PV modules with and without mirror augmentation as measured in the outdoor test facility. The modules are mounted at $55^{\circ}$ and the mirror mounted at $0^{\circ}$. This configuration is expected to increase augmentation during the winter when sun elevation angles are lower. The I-V curve in this figure is only one snapshot of a very specific time. The power output on the MAPV panel is $223.8 \mathrm{~W}$ compared to $199.4 \mathrm{~W}$ of the non-augmented panel. It is obvious that 3 steps in the MAPV I-V curve indicate that the module bypass diodes are operating indicating that the light is non-uniform across the panel. In addition to the I-V snapshot, power production data was recorded for a 2-week period in January. Over this time-limited period of study the MAPV system produced $26.2 \%$ more power than an equivalent non-augmented module.

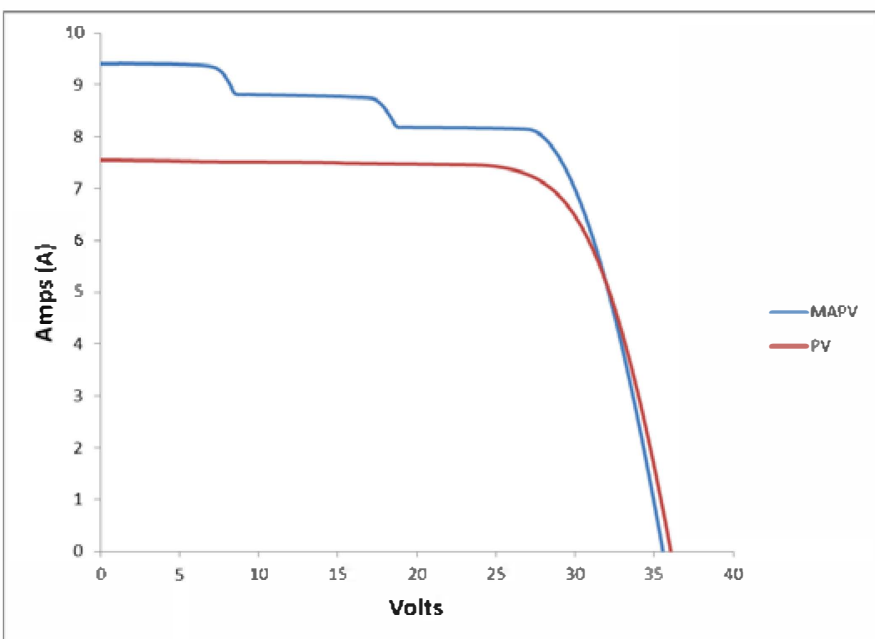

Figure 7. Performance Comparison on 8th January: MAPV vs. Non-augmented

\section{CONCLUSION}

Photovoltaic electricity has the potential to serve as a competitive and efficient energy source in the future. However, the prime cost of this technology is still higher than nuclear, thermal and wind power. One simple and effective way to drive down the cost of PV electricity is to combine reflectors and PV panels in order to harvest more light from the modules. In this study, an optimum configuration design is used to analyze the non-uniformity issue for a fixed (non-tracked) MAPV system. The nonuniformity was caused by both shadowing effects and mirror augmentation. The mirror augmented systems also experienced end effects during which the panel was not fully augmented because of inadequate mirror lengths. By modifying the length of mirror, we can remove the endeffect in the mirror augmented systems. The optical analysis helps us to predict the differential degradation rates on modules. The optical analysis and I-V curve both prove that the MAPV system has higher power output compared to a non-augmented PV system.

\section{ACKNOWLEDGMENT}

The authors gratefully acknowledge the contributions of research members in VUV-Lab and Replex Plastics for their work on the original version of this document.

\section{BIOGRAPHIES}

Wei-Chun Lin is currently a Ph.D. graduate student at Case

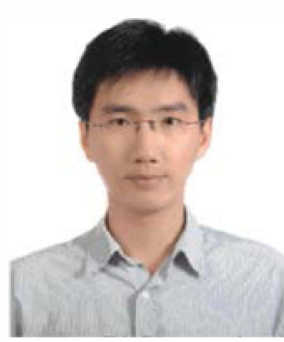

Western Reserve University. His previous working experience focused on surface analysis of organic materials at Academia Sinica in Taiwan. Before entering Case Western Reserve at 2010, he authored three journal articles and co-authored several papers. His current research focuses on optimizing mirror- augmentation photovoltaic modules and extending its reliability and service lifetime.

David A. Hollingshead is a Project Engineer at Replex

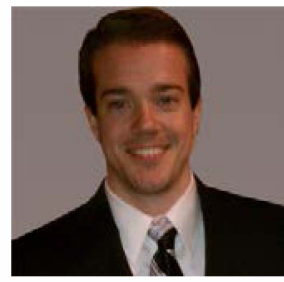
Plastics. He recently graduated from The Ohio State University with Bachelor of Science and Master of Science degrees in Electrical Engineering. Dave has been working on the solar effort at Replex since April 2011. His work at Replex has focused on system modeling, performance testing and data acquisition, vacuum metallization, protective coatings, and electrical interconnect design.

Kara A. Shell is currently a Project Engineer at Replex

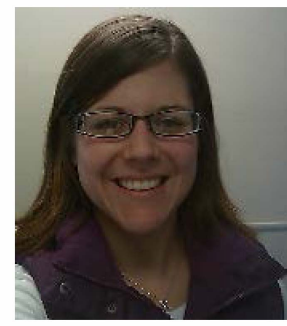
Plastics. She recently graduated from The Ohio State University with a Bachelor of Science Magna Cum Laude and a Master of Science in Mechanical Engineering. Her work at Replex involves the use of such 
advanced tools as 3D CAD Modeling, Integrated CAD/CAM programming, Finite Element Analysis, and Optical Ray Tracing. Shell is involved in all aspects of the product design process, from concept to prototype construction to the design and execution of experiments to evaluate prototypes.

Joseph Karas is currently an undergraduate student at Case

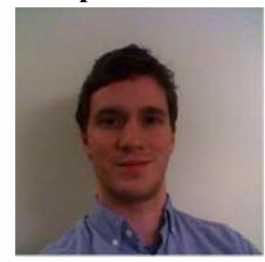

Western Reserve University studying Polymer Science \& Engineering. His current work focuses on the determination of power degradation rates in mirroraugmented photovoltaic systems.

Scott Brown is a Manufacturing Engineer at Replex with over

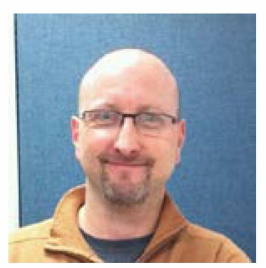

20 years in Engineering and Manufacturing specializing in Manufacturing methods and processes. Brown has been involved in the solar effort at Replex Plastics since February 2009, bringing to bear previous experience in thermoforming, metallizing, and coatings. He is currently doing work on mirror design and manufacturing methods; material, component and system durability testing; and system design and performance testing.

Mark A. Schuetz is the Founder and President of Replex

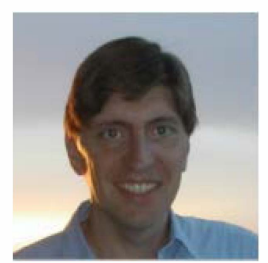
Plastics. He holds a Bachelor of Science in Mechanical Engineering and Master of Science from the Massachusetts Institute of Technology. Founded in 1991, Replex has become a world leader in the manufacture of convex acrylic mirrors, polycarbonate playground domes, and thermoformed closed circuit TV domes.

Yang Hu is currently a Ph.D. graduate student at Case

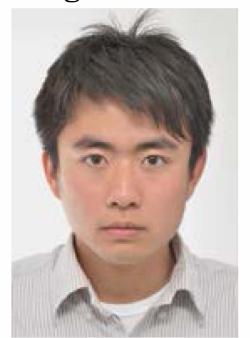
Western Reserve University in the Department of Materials Science and Engineering. He received a Bachelor of Engineering in Opto-electronics Science and Technology from Tianjin University, China. His current research focus on outdoor test of PV modules and PV power degradation.

Dr. Roger French (F. Alex Nason Professor, Department of Materials Science and Engineering) joined CWRU in August 2010 after 24 years of conducting basic research and product

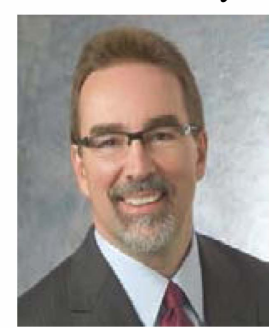
development in DuPont's Central Research. He is the director of the Solar Durability and Lifetime Extension Center at CWRU. Dr. French has a broad experience in developing and commercializing optical materials for many different applications and in optimizing these materials for improved radiation durability and lifetime. Dr. French is also a nationally recognized expert in Lifetime and Degradation Science (L\&DS) for commercial applications, evidenced by his work on attenuating phase shift photomasks, fluoropolymer pellicles for photolithography, immersion lithography imaging fluids, and materials for concentrating photovoltaic systems. He has 22 issued patents and more than 145 publications.

\section{REFERENCES}

${ }^{1}$ R. H. French, M. P. Murray, W. C. Lin, K. A. Shell, S. A. Brown, M. A. Schuetz, R. J. Davis, "Solar radiation durability of materials components and systems for low concentration photovoltaic systems", IEEE Energytech, Cleveland, USA, May 2011.

${ }^{2}$ The information of solar mirror was provided by Replex Plastics Corp., Mount Vernon, OH [online] (2012, Feb.). Available: http://www.replex.com/?id=applications\&pg=solar

3 Ibrahim Reda, and Afshin Andreas, "Solar position algorithm for solar radiation applications," National Renewable Energy Laboratory, Golden, Colorado, Tech. Rep. NREL/TP-560-34302, Revised Jan. 2008.

${ }^{4}$ The information of CS-6P was provided by CSI Corp., Ontario, Canada [online] (2012, Feb.). Available: http://solarinstallco.com/images/cs6p200.pdf

${ }^{5}$ The information of Multi-tracer was provided by Daystar Corp., Las Cruces, NM [online] (2012, Feb.). Available: http://www.zianet.com/daystar/multitracer.html

${ }^{6}$ The information of Pyranometer CMP6 was provided by Kipp\&Zonen Corp., Netherlands [online] (2012, Feb.). Available: http://www.kippzonen.com/?product/1251/CMP+6.aspx 